\title{
Rheumanomics: Addressing Scarcity and Need in Rheumatologic Care
}

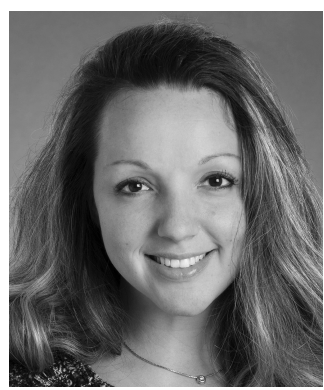

They say that necessity is the mother of invention. And the delivery of rheumatologic care is in need of mothering.

In this month's Journal, Rostom and colleagues ${ }^{1}$ describe the use and benefits of an innovative eConsult service, developed to improve access to rheumatologic care in the Champlain region in eastern Ontario, Canada. They categorized the 225 eConsults directed to rheumatology over a span of nearly 4 years, between mid-2011 and early 2015, according to type of question posed and the effect of the interaction on face-to-face referral rates. The group found that referrals for osteoporosis, polyarthralgias, and polyarthritis were the most common, and that consults centered around drug treatment and diagnosis. The strengths cited were limited additional demand placed on a strained system, efficiency of response, referral avoidance, and high user satisfaction.

With aging baby boomers, population expansion, migration, and the advent of complex rheumatologic therapies, there is a burgeoning need for rheumatologic care. The problem is one of supply and demand: either there is a shortage of rheumatologists or there are too many potential rheumatology patients. The solution is "simple": What do we need? More rheumatologists. When do we want them? Now.

Let us pause, however, to consider the truly scarce resource here: what we really need is increased rheumatologic acumen, systemwide, that can then be dispensed as rheumatologic assessments. Some clinical issues require multiple assessments, others need fewer. And while rheumatologists are acumen-dense and can provide a large number of assessments for the widest range of rheumatologic issues, they are limited and costly resources, and the supply of rheumatologists is fairly static.

Demand for rheumatology assessments currently vastly exceeds supply. In a public healthcare system, this means longer wait times that will only get longer unless supply is somehow increased. The projected increased burden of rheumatic diseases makes the prospect of decreased demand implausible for the foreseeable future if things do not change ${ }^{2}$. One Canadian study revealed that $31 \%$ of patients wait 3 months or longer for consultation, nationwide ${ }^{3}$. Rheumatologists are not achieving the wait-time benchmarks for inflammatory arthritides and autoimmune diseases ${ }^{4}$. This does not even address the hidden demand from patients who ought to have a rheumatologic assessment, but cannot access one, either because they do not have access to primary care or because their need is unrecognized (either by the patient or by the assessment gatekeeper, i.e., the referral source).

The supply problem can only be overcome by increasing rheumatology assessments to better match demand. Options to bring this about are (1) decrease time required to provide a rheumatology assessment, and/or (2) increase the number of rheumatology assessments possible in the system/unit time.

There is a practical lower limit to the time in which a conventional rheumatologic assessment can be safely and competently completed. It is thus unlikely that appreciable gains will be made from lighting a fire under practicing rheumatologists, who have likely already optimized their personal efficiency. There is a practical upper limit to the number of additional rheumatologists that can be incorporated into a system facing similarly dire supply-demand disparity across specialties. In Ontario, where Rostom's eConsult service was implemented, the number of rheumatologists has remained static at 160 between 1996 and 2010, and rheumatoid arthritis (RA) prevalence has doubled, from $0.5 \%$ to $0.9 \%$ over the same time frame ${ }^{5}$. The study also found that the RA burden was increased in northern regions compared to urban areas, inversely proportional to the concentration of practicing rheumatologists.

We are thus left with 1 option: to increase the system's capacity for rheumatologic assessment. This would ideally leverage resources already in place. A combination of rheumatologists, primary care providers, and extended-role practitioners could achieve better triage of inflammatory arthritis by harnessing technology to reach patients living more remotely. After all, many hands make light work, and rheumatologists' hands are not best suited to solve this problem independently. While perhaps intuitive, the literature suggests that available resources are not yet optimized.

Rostom's eConsults service demonstrates the elegant efficiency that can be afforded by leveraging technology to increase capacity for rheumatologic assessments. In addition

See Electronic consultation and rheumatologic care access, page 137 
to the value of 225 assessments completed, it facilitates learning from the interaction with the eConsulting rheumatologist. And yet this service has not yet been widely implemented.

Rheumatologists' expertise is best used in the diagnosis and management of inflammatory arthritis and autoimmune diseases, which does not appear to be reflected in referral patterns from primary care providers. A contemporary and enlightening study by Widdifield and colleagues found an equal proportion of referrals made for osteoarthritis (OA) and systemic inflammatory rheumatic diseases, with each accounting for about one-third of the consultations requested ${ }^{6}$. Herein lies an opportunity, as many rheumatologists believe OA care falls predominantly within the realm of primary care. The Arthritis Alliance of Canada has developed an OA toolkit, providing therapeutic decision support for OA management ${ }^{7}$. Its uptake and effect on referral patterns will be of interest in the years to come.

While benchmarks for time to referral to rheumatologic consultation are unmet, the Widdifield, et al study suggests that this is not the most significant bottleneck from symptom onset to management ${ }^{6}$. The study further dissects the median 327 days from RA symptom onset to rheumatologist consultation, revealing that the majority of the time was spent preconsultation (median 173 days from symptom onset to primary care visit, and median 115 days from first mention at primary care to rheumatology referral). Primary care providers can be effective allies, and can deliver rheumatologic assessment if armed with the rheumatologic knowledge to ask high-yield questions and appreciate patterns that raise rheumatologic red flags. This is especially true if an organic community of practice can be built, wherein primary care providers feel supported in co-management by the rheumatologist. This arrangement is supported by the Extension for Community Healthcare Outcomes (ECHO) Project, which was developed to improve access to high-quality care for patients with complex health issues by engaging primary care providers through weekly videoconferencing ${ }^{8}$. While referrals to rheumatologists may paradoxically rise with increased primary care awareness, the nature of referrals may evolve to better address previously unmet needs and to improve outcomes.

I would be remiss if I did not stress that specialized physical examination is central to rheumatologic diagnosis. Extendedrole providers, typically allied health professionals with additional training in rheumatology, including physical examination, have an important position. These practitioners (such as the Advanced Clinician Practitioner in Arthritis Care graduates in Ontario) have the capability to act as intermediaries between primary care and rheumatologists, improve triage, and ultimately lead to more timely rheumatologic management ${ }^{9}$.

We cannot solve this problem alone. These fledgling programs, implemented and measured systematically, and adapted to local context, hold the promise of optimizing the care we deliver. There have been calls to measure and improve the delivery of care to rheumatology patients ${ }^{10}$.
Responses to date prove there is more than 1 way to approach this issue. The next step, then, is to rally these multidisciplinary troops, and unify our next steps forward. There is an opportunity here for the whole to be greater than the sum of its parts, which, if realized, has the potential to bridge the supply-demand gap and simultaneously revolutionize care delivery to patients with rheumatic diseases.

AMANDA STEIMAN, MD, MSc, $\operatorname{FRCP}(C)$,

Staff Rheumatologist,

Division of Rheumatology,

Sinai Health System/

University Health Network,

Rebecca MacDonald Centre for

Arthritis and Autoimmune Disease.

Address correspondence to Dr. A. Steiman, Sinai Health

System/University Health Network, Rebecca MacDonald Centre for

Arthritis and Autoimmune Disease, 60 Murray St., Suite 2-223,

Box 10, Toronto, Ontario M5T 3L9, Canada.

E-mail: amanda.steiman@sinaihealthsystem.ca

\section{REFERENCES}

1. Rostom K, Smith CD, Liddy C, Afkham A, Keely E. Improving access to rheumatologists: use and benefits of an electronic consultation service. J Rheumatol 2018;45:137-40.

2. Smith E, Hoy DG, Cross M, Vos T, Naghavi M, Buchbinder R, et al. The global burden of other musculoskeletal disorders: estimates from the Global Burden of Disease 2010 study. Ann Rheum Dis 2014;73:1462-9.

3. Carriere G, Sanmartin C. Waiting time for medical specialist consultations in Canada, 2007. Health Rep 2010;21:7-14.

4. Wait-time benchmarks for rheumatology. Canadian Rheumatology Association; 2015. [Internet. Accessed October 26, 2017.] Available from: www.waittimealliance.ca/wp-content/uploads/2014/05/ Wait-Time-Benchmarks-for-Rheumatology-FINAL.pdf

5. Widdifield J, Paterson JM, Bernatsky S, Tu K, Thorne JC, Ahluwalia $\mathrm{V}$, et al. The rising burden of rheumatoid arthritis surpasses rheumatology supply in Ontario. Can J Public Health 2013;104:e450-e455.

6. Widdifield J, Bernatsky S, Thorne JC, Bombardier C, Jaakkimainen $\mathrm{L}$, Wing L, et al. Wait times to rheumatology care for patients with rheumatic diseases: a data linkage study of primary care electronic medical records and administrative data. CMAJ Open 2016;4: E205-12.

7. Arthritis Alliance of Canada, the Centre for Effective Practice, the College of Family Physicians of Canada. Osteoarthritis Tool. 2017. [Internet. Accessed October 26, 2017.] Available from: http:// arthritisalliance.ca/images/OA_Tool_Final_July_24_2017_ENG.pdf

8. Arora S, Thornton K, Murata G, Deming P, Kalishman S, Dion D, et al. Outcomes of treatment for hepatitis $\mathrm{C}$ virus infection by primary care providers. N Engl J Med 2011;364:2199-207.

9. Lundon K, Shupak R, Schneider R, Herold McIlroy J. Development and early evaluation of an inter-professional post-licensure education programme for extended practice roles in arthritis care. Physiother Can 2011 Winter;63:94-103.

10. Barber CE, Marshall DA, Mosher DP, Akhavan P, Tucker L, Houghton K, et al; Arthritis Alliance of Canada Performance Measurement Development Panel. Development of system-level performance measures for evaluation of models of care for inflammatory arthritis in Canada. J Rheumatol 2016;43:530-40.

J Rheumatol 2018;45:1-2; doi:10.3899/jrheum.171179 